\title{
ASSOCIATED PRIMES OF LOCAL COHOMOLOGY AFTER ADJOINING INDETERMINATES. PART 2: THE GENERAL CASE
}

\author{
HANNAH ROBBINS
}

\begin{abstract}
Let $A$ be a domain finitely generated as an algebra over a field, $k$ of characteristic zero, $R=$ $A\left[t_{1}, \ldots, t_{\ell}\right]$ or $A\left[\left[t_{1}, \ldots, t_{\ell}\right]\right]$, and $I$ an ideal of $R$. If $A$ has a resolution of singularities, $Y_{0}$, which is the blowup of $A$ along an ideal of depth at least 2 and is covered by a finite number of open affines with $H^{j}\left(Y_{0}, \mathcal{O}_{Y_{0}}\right)$ of finite length over $A$ for $j>0$, we prove that $\operatorname{Ass}_{R} H_{I}^{i}(R)$ is finite for every $i$. In particular, this holds when $A$ is a finite-dimensional normal domain with an isolated singularity which is a finitely generated algebra over a field of characteristic 0 .
\end{abstract}

1. Introduction. The problem of when the assassinators of local cohomology modules are finite has been widely studied for some time. Many mathematicians have looked at the general case where one considers the local cohomology of any module, but others have restricted their focus to the case where we only attempt to control the local cohomology of the ring itself. In this setting, the case of regular rings is particularly nice. If $S$ is a regular ring of equal characteristic $p>0$, Huneke and Sharp showed in [3] that $\operatorname{Ass}_{S} H_{I}^{i}(S)$ is always finite. In characteristic 0, Lyubeznik used the theory of $D$-modules in [4] to show that $\operatorname{Ass}_{S} H_{I}^{i}(S)$ is finite for regular domains, $S$, which are finite algebras over a field of characteristic 0 .

My last paper [7] began exploring a way to extend these results on the finiteness of $\operatorname{Ass}_{S} H_{I}^{i}(S)$ to polynomial or power series extensions of a ring, whose resolution of singularities is covered by regular rings of the type studied by Lyubeznik. This allowed the use of techniques from algebraic geometry to link the good behavior of the regular rings covering the resolution of singularities to the polynomial or power series extensions of the base ring. In that paper, I considered base

2010 AMS Mathematics subject classification. Primary 13D45.

Keywords and phrases. Associated primes, local cohomology.

Received by the editors on March 11, 2015. 
rings whose resolutions of singularities can be covered by two or three open affines, but here we extend those ideas to the case of a base ring whose resolution of singularities is covered by any finite number of open affines.

The main result of this paper is Theorem 3.1. In it, we take a Noetherian domain which is a finite algebra over a field of characteristic zero as our base ring, $A$. We form $R$ by adjoining finitely many variables to $A$, either as polynomials or power series. If $A$ has a blowup, $Y_{0}$, along an ideal of depth at least 2 which is covered by finitely many affine patches so that all higher cohomology of its structure sheaf $\mathcal{O}_{Y_{0}}$ has finite length over $A$, then Theorem 3.1 shows that $\operatorname{Ass}_{R} H_{I}^{i}(R)$ is always finite.

2. New material. We start by establishing some basic notation. Let $k$ be a field of characteristic 0 . Let $A$ be a Noetherian domain finitely generated as a $k$-algebra, which has a resolution of singularities, $Y_{0}$, that is the blowup of $A$ along an ideal of depth at least 2 and has an open cover by affine patches $U_{1}, \ldots, U_{m}$ so that $H^{j}\left(Y_{0}, \mathcal{O}_{Y_{0}}\right)$ has finite length over $A$ for all $j>0$. We will let $S_{p}$ be the regular ring corresponding to $U_{p}$.

Let $R=A\left[t_{1}, \ldots, t_{\ell}\right]$ or $A\left[\left[t_{1}, \ldots, t_{\ell}\right]\right]$, and let $I$ be any ideal of $R$. Note that our proofs will be done in the polynomial case, but the same proofs work for power series.

Our first step in controlling the assassinators of the local cohomology modules of $R$ with respect to $I$ is to create two collections of double complexes.

Take generators of $I$ so that $I=\left(f_{1}, \ldots, f_{n}\right) \subset R$. Let $A_{0}^{\bullet \bullet}$ be the double complex formed by tensoring the complex used to compute the sheaf cohomology of $\mathcal{O}_{Y}$,

$$
0 \longrightarrow \oplus S_{p} \longrightarrow \oplus S_{p q} \longrightarrow \cdots \longrightarrow S_{1 \cdots m} \longrightarrow 0,
$$

with the complex used to compute local cohomology of $R$ with respect to $I$,

$$
0 \longrightarrow R \longrightarrow \oplus R_{f_{i}} \longrightarrow \oplus R_{f_{i} f_{j}} \longrightarrow \cdots \longrightarrow R_{f_{1} \cdots f_{n}} \longrightarrow 0 .
$$

Thus, $A_{0}^{\bullet \bullet}$ is the complex given below, with the 0th column on the left and the $(m-1)$ st column on the right. 


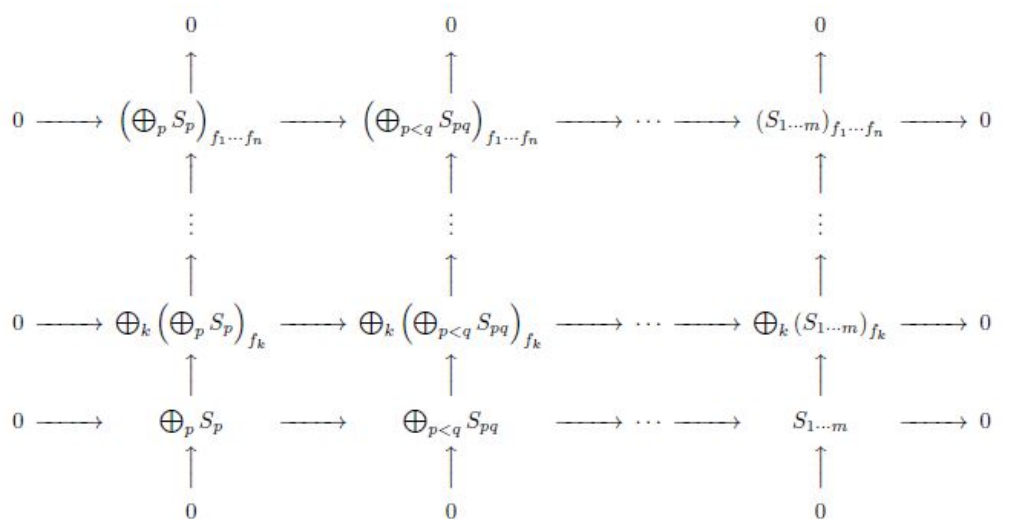

We filter this complex by subcomplexes $A_{0}^{\bullet \bullet}\langle k\rangle$, which are simply $A_{0}^{\bullet \bullet}$ with the first $k$ rows replaced by zeros. Let $E_{0}$ be the associated graded complex with respect to this filtration, so that $E_{1}$ is the total complex of $A_{1}^{\boldsymbol{\bullet} \bullet}$ where $A_{1}^{i j}=\bigoplus H^{j}\left(Y, \mathcal{O}_{Y}\right)_{f_{k_{1}} \cdots f_{k_{i}}}$, the horizontal maps are 0 , and the vertical maps are induced by the vertical maps of $A_{0}^{\mathbf{\bullet}}$.

Here, $d^{1}: A_{1}^{i j} \rightarrow A_{1}^{i+1, j}$, so we are simply taking cohomology along each column. This means $E_{2}$ is the total complex of $A_{2}^{\bullet \bullet}$, where $A_{2}^{i j}=H_{I}^{i}\left(H^{j}\left(Y, \mathcal{O}_{Y}\right)\right)$.

We can continue this process to create a set of double complexes,

$$
A_{1}^{\bullet \bullet}, A_{2}^{\bullet \bullet}, \ldots, A_{k}^{\bullet \bullet}, \ldots, A_{m}^{\bullet \bullet},
$$

where the differential at stage $k$ is $d^{k}: A_{k}^{i j} \rightarrow A_{k}^{i+k, j-(k-1)}$.

Lemma 2.1. $A_{k}^{i j}$ is holonomic for all $k \geq 2$ and all $j>0$.

Proof. (By induction on $k$.) Our base case is $k=2$, so we are looking at $A_{2}^{i j}=H_{I}^{i}\left(H^{j}\left(Y, \mathcal{O}_{Y}\right)\right)$, where $j>0$.

To show this module is holonomic, we use $D$-module methods. Let $D=k\left[t_{1}, \ldots, t_{\ell}, \partial_{1}, \ldots, \partial_{\ell}\right]$, where $\partial_{i}$ is differentiation with respect to $t_{i}$. We can extend the action of $k\left[t_{1}, \ldots, t_{\ell}\right]$ on $R$ to an action of $D$ by setting $\partial_{i}(a)=0$ for all $a \in A$. 
As each $S_{p}$ is generated over $R$ by finitely many fractions of elements from $R$, we can define an action of $D$ on them via the quotient rule. This $D$ action extends to all localizations and is compatible with localization maps, making every module in $A_{0}^{\bullet \bullet}$ a $D$-module and all its maps $D$ module maps. In fact, all the modules and maps in every $A_{k}^{\bullet \bullet}$ will have a $D$-module structure.

Because $H^{j}\left(Y_{0}, \mathcal{O}_{Y_{0}}\right)$ has finite length over $A$ for $j>0$, as a module over $k$ it is a finite dimensional $k$-vector space. This means $H^{j}\left(Y, \mathcal{O}_{Y}\right) \cong H^{j}\left(Y_{0}, \mathcal{O}_{Y_{0}}\right) \otimes_{k} k\left[t_{1}, \ldots, t_{m}\right]$ is just a direct sum of copies of $k\left[t_{1}, \ldots, t_{m}\right]$, which is holonomic by [4, Property 2.2 (a) and Remark 2.9]. Thus, whenever $j>0, A_{2}^{i j}=H_{I}^{i}\left(H^{j}\left(Y, \mathcal{O}_{Y}\right)\right)$ is the local cohomology of a holonomic $D$-module and therefore is also holonomic by $[4$, Property $2.2(\mathrm{~d})]$.

Now assume that $A_{k}^{i j}$ is holonomic for every $j>0$.

We know $A_{k+1}^{i j}$ is the cohomology of the map $d^{k}$ at the $i j$ th spot. Since $d^{k}$ maps up $k$ rows and left $k-1$ columns, we have

$$
A_{k+1}^{i j}=\frac{\operatorname{ker}\left(d^{k}: A_{k}^{i j} \rightarrow A_{k}^{i+k, j-(k-1)}\right)}{d^{k}\left(A_{k}^{i-k, j+(k-1)}\right)} .
$$

By our induction hypothesis, $A_{k}^{i j}$ is holonomic. Since the kernel of $d^{k}$ in the numerator is a $D$-submodule of $A_{k}^{i j}$, this means it is also holonomic by [4, Property 2.2 (c)].

Similarly, since $j+(k-1)>0$, we have $A_{k}^{i-k, j+(k-1)}$ holonomic by our induction hypothesis. Since $d^{k}$ is a $D$-module map, the image in the denominator is holonomic by [4, Property 2.2 (c)].

Therefore, $A_{k+1}^{i j}$ is a quotient of holonomic of $D$-modules and hence is holonomic by [4, Property 2.2 (c)].

Lemma 2.2. If $k \geq 3$ and $\operatorname{Ass}_{R} A_{k}^{i 0}$ is finite, then $\operatorname{Ass}_{R} A_{k-1}^{i 0}$ is also finite.

Proof. We know $A_{k}^{i 0}=\frac{A_{k-1}^{i 0}}{d^{k-1}\left(A_{k-1}^{i-(k-1), k-2}\right)}$. Since $k \geq 3$, we have $A_{k-1}^{i-(k-1), k-2}$ holonomic by Lemma 2.1. This means that its image under $d^{k-1}$ is also holonomic, so $\operatorname{Ass}_{R} d^{k-1}\left(A_{k-1}^{i-(k-1), k-2}\right)$ is finite. 
Since $\operatorname{Ass}_{R} A_{k}^{i 0}$ is finite, we see $A_{k-1}^{i 0}$ has a finite assassinator after a quotient by a module which also has a finite assassinator. Thus, $\operatorname{Ass}_{R} A_{k-1}^{i 0}$ is finite as claimed.

We can also filter our original double complex $A_{0}^{\bullet \bullet}$ by columns instead of rows. We will call the double complexes from this new filtration $B_{k}^{\bullet \bullet}$ s to avoid confusion. Here, $B_{0}^{\bullet \bullet}=A_{0}^{\bullet \bullet}$, and $B_{0}^{\bullet \bullet}\langle k\rangle$ is just $B_{0}^{\bullet \bullet}$ with the first $k$ columns replaced by zeros. Similar to our filtration by rows, let $E_{0}$ be the associated graded complex with respect to this new filtration. This makes $E_{1}$ the total complex of $B_{1}^{\bullet \bullet}$ where $B_{1}^{i 0}=H_{I}^{i}\left(\oplus S_{k}\right), B_{1}^{i 1}=H_{I}^{i}\left(\oplus S_{k \ell}\right)$ and so on up to $B_{1}^{i, m-1}=H_{I}^{i}\left(S_{1 \cdots m}\right)$. All vertical maps are 0 , and the horizontal maps are induced by the corresponding maps in $B_{0}^{\bullet \bullet}$.

Here, $d^{1}: E_{1}^{p q} \rightarrow E_{1}^{p, q+1}$, so, to get $E_{2}$, we are taking cohomology along each row. The $i$ th row is the Cech complex which computes cohomology of the sheaf $\mathcal{H}_{I}^{i}\left(\mathcal{O}_{Y}\right)$ with respect to the cover of $Y$ by $U_{1}, \ldots, U_{m}$. Thus, $E_{2}$ is the total complex of $B_{2}^{\bullet \bullet}$, where $B_{2}^{i j}=$ $H^{j}\left(Y, \mathcal{H}_{I}^{i}\left(\mathcal{O}_{Y}\right)\right)$ and the differential is $d^{2}: B_{2}^{i j} \rightarrow B_{2}^{i-1, j+2}$.

As with filtration by rows, we can continue this to create a set of double complexes

$$
B_{1}^{\bullet \bullet}, B_{2}^{\bullet \bullet}, \ldots, B_{k}^{\bullet \bullet}, \ldots, B_{m-1}^{\bullet \bullet},
$$

where our differential is $d^{k}: B_{k}^{i j} \rightarrow B_{k}^{i-(k-1), j+k}$.

Lemma 2.3. $\operatorname{Ass}_{R} B_{k}^{i 0}$ is finite for all $k \geq 2$.

Proof. (By induction on $k$.) Our base case is $k=2$, where $B_{2}^{i 0}=$ $H^{0}\left(Y, \mathcal{H}_{I}^{i}\left(\mathcal{O}_{Y}\right)\right)$.

We know $H^{0}\left(Y, \mathcal{H}_{I}^{i}\left(\mathcal{O}_{Y}\right)\right) \subseteq H_{I}^{i}\left(S_{1}\right) \oplus \cdots \oplus H_{I}^{i}\left(S_{m}\right)$, where the $S_{p}$ are all regular. This means $\operatorname{Ass}_{S_{p}} H_{I}^{i}\left(S_{p}\right)$ is finite for $1 \leq p \leq m$. Since the associated primes of $H_{I}^{i}\left(S_{p}\right)$ over $R$ will be restrictions of the associated primes over $S_{p}$, we conclude that $\operatorname{Ass}_{R} B_{2}^{i 0}$ is finite.

Now assume that $\operatorname{Ass}_{R} B_{k}^{i 0}$ is finite.

We know that $B_{k+1}^{i 0}$ is the cohomology of the map $d^{k}$, which maps down $k-1$ rows and right $k$ columns, at the $i 0$ th spot. The image of $d^{k}$ is 0 at this spot, so we have

$$
B_{k+1}^{i 0}=\operatorname{ker}\left(d^{k}: B_{k}^{i 0} \longrightarrow B_{k}^{i-(k-1), k}\right) \subseteq B_{k}^{i 0} .
$$


Since $\operatorname{Ass}_{R} B_{k}^{i 0}$ is finite by our induction hypothesis, this means $\operatorname{Ass}_{R} B_{k+1}^{i 0}$ is finite as well, and we are done.

3. Main result. Our main theorem shows that the local cohomology of our polynomial or power series ring $R$ with respect to any ideal $I \subseteq R$ has only finitely many associated primes. For ease of reference, all our assumptions will be restated in the statement of Theorem 3.1.

We will act in the proof as if the number of affine patches covering the resolution of singularities $Y_{0}$ is three or more. For the case of two affine patches, see [7, Theorem 2].

Theorem 3.1. Let $A$ be a domain, finitely generated as an algebra over a field, $k$ of characteristic 0 , and let $R=A\left[t_{1}, \ldots, t_{\ell}\right]$ or $A\left[\left[t_{1}, \ldots, t_{\ell}\right]\right]$. If $A$ has a resolution of singularities, $Y_{0}$, which is the blowup of $A$ along an ideal of depth at least 2 and has an open affine cover by $U_{1}, \ldots, U_{m}$, where $H^{j}\left(Y_{0}, \mathcal{O}_{Y_{0}}\right)$ has finite length over $A$ for all $j>0$, then $\operatorname{Ass}_{R} H_{I}^{i}(R)$ is finite for any $i$ and any ideal $I \subset R$.

Proof. Consider our double complexes $A_{k}^{\bullet \bullet}$. From [7, Lemma 1], we get $H^{0}\left(Y, \mathcal{O}_{Y}\right) \cong R$. This means $A_{2}^{i 0}=H_{I}^{i}\left(H^{0}\left(Y, \mathcal{O}_{Y}\right)\right) \cong H_{I}^{i}(R)$, so we are really just interested in controlling the associated primes of $A_{2}^{i 0}$ for every $i$.

To do this, let us consider the last double complex in this sequence: $A_{m}^{\bullet \bullet}$. Our differential here is $d^{m}: A_{m}^{i j} \rightarrow A_{m}^{i+m, j-(m-1)}$. There are only $m$ nonzero columns, so this map is only nontrivial if $j=m-1$, i.e., $d^{m}: A_{m}^{i, m-1} \rightarrow A_{m}^{i+m, 0}$. This means that our associated graded complex $E_{m+1}^{\bullet \bullet}$ has

$$
\begin{aligned}
& E_{m+1}^{i 0}=\frac{A_{m}^{i 0}}{d^{m}\left(A_{m}^{i-m, m-1}\right)}, \\
& E_{m+1}^{i j}=A_{m}^{i j} \quad \text { for all } 1 \leq j \leq m-2,
\end{aligned}
$$

and

$$
E_{m+1}^{i, m-1}=\operatorname{ker}\left(d^{m}: A_{m}^{i, m-1} \longrightarrow A_{m}^{i+m, 0}\right) .
$$

Since we have $d^{r} \equiv 0$ for all $r \geq m+1$, meaning that $E_{m+1}=E_{\infty}$, we get an exact sequence for every $i$, 


$$
0 \longrightarrow E_{\infty}^{i-m, m-1} \longrightarrow A_{m}^{i-m, m-1} \longrightarrow A_{m}^{i 0} \longrightarrow E_{\infty}^{i 0} \longrightarrow 0
$$

where the middle map is $d^{m}$.

Letting $\mathcal{T}^{\bullet}$ denote the total complex of $E_{\infty}$, then $\mathcal{T}^{i}=E_{\infty}^{i 0} \oplus E_{\infty}^{i-1,1} \oplus$ $\cdots \oplus E_{\infty}^{i-(m-1), m-1}$, gives us an exact sequence

$$
\mathcal{T}^{i-1} \longrightarrow A_{m}^{i-m, m-1} \longrightarrow A_{m}^{i 0} \longrightarrow \mathcal{T}^{i}
$$

where the first map is a projection onto $E_{\infty}^{i-m, m-1}$, the middle map is $d^{m}$ and the last map comes from the projection of $A_{m}^{i 0}$ onto $E_{\infty}^{i 0} \subseteq \mathcal{T}^{i}$.

We can therefore think of $A_{m}^{i 0}$ as the center of the short exact sequence

$$
0 \longrightarrow d^{m}\left(A_{m}^{i-m, m-1}\right) \longrightarrow A_{m}^{i 0} \longrightarrow \operatorname{im}\left(A_{m}^{i 0}\right) \longrightarrow 0,
$$

where $\operatorname{im}\left(A_{m}^{i 0}\right)$ is the image of $A_{m}^{i 0}$ inside $\mathcal{T}^{i}$.

Since $m>3$, and hence $m-1>0$, Lemma 2.1 implies that $A_{m}^{i-m, m-1}$ is holonomic. This means that its image under the $D$-module map $d^{m}$ is also holonomic, and hence, $d^{m}\left(A_{m}^{i-m, m-1}\right)$ has finitely many associated primes. Thus, to show $\operatorname{Ass}_{R} A_{m}^{i 0}$ is finite, we need only control $\operatorname{Ass}_{R} \operatorname{im}\left(A_{m}^{i 0}\right)$.

To do this, we look at the $B_{k}^{\bullet \bullet}$ s. The last nonzero such complex is $B_{m-1}^{\bullet \bullet}$, where our differential is $d^{m-1}: B_{m-1}^{i j} \rightarrow B_{m-1}^{i-(m-2), j+(m-1)}$. Since there are only $m$ nonzero columns, the differential is the zero map except for $d^{m-1}: B_{m-1}^{i 0} \rightarrow B_{m-1}^{i-(m-2), m-1}$. This means that $E_{m}$ is the total complex of $B_{m}^{\bullet \bullet}$, where

$$
\begin{aligned}
& B_{m}^{i 0}=\operatorname{ker}\left(d^{m-1}: B_{m-1}^{i 0} \rightarrow B_{m-1}^{i-(m-2), m-1}\right), \\
& B_{m}^{i j}=B_{m-1}^{i j} \quad \text { for all } 1 \leq j \leq m-2,
\end{aligned}
$$

and

$$
B_{m}^{i, m-1}=\frac{B_{m-1}^{i, m-1}}{d^{m-1}\left(B_{m-1}^{i+(m-2), 0}\right)} .
$$

Because $d^{m} \equiv 0$, we have $E_{\infty}=E_{m}$. For each $i$, this gives us the short exact sequence 


$$
0 \longrightarrow E_{\infty}^{i 0} \longrightarrow B_{m-1}^{i 0} \longrightarrow B_{m-1}^{i-(m-2), m-1} \longrightarrow E_{\infty}^{i-(m-2), m-1} \longrightarrow 0,
$$

where the middle map is $d^{m-1}$.

Again, letting $\mathcal{T}^{\bullet}$ be the total complex of $E_{\infty}$, we have

$$
\mathcal{T}^{i}=E_{\infty}^{i 0} \oplus E_{\infty}^{i-1,1} \oplus \cdots \oplus E_{\infty}^{i-(m-1), m-1} .
$$

This means that $\mathcal{T}^{i}$ maps onto $E_{\infty}^{i 0}=B_{m}^{i 0}$ via the short exact sequence

$$
0 \longrightarrow B_{m}^{i-1,1} \oplus \cdots \oplus B_{m}^{i-(m-1), m-1} \longrightarrow \mathcal{T}^{i} \longrightarrow B_{m}^{i 0} \longrightarrow 0 .
$$

Since $A_{m}^{i 0}$ maps to $\mathcal{T}^{i}$, its image in $\mathcal{T}^{i}$ also maps to $B_{m}^{i 0}$, and the kernel of this mapping is the intersection of the images of $\operatorname{im}\left(A_{m}^{i 0}\right)$ and $B_{m}^{i-1,1} \oplus \cdots \oplus B_{m}^{i-(m-1), m-1}$ inside $\mathcal{T}^{i}$.

Lemma 3.2. The images of $A_{m}^{i 0}$ and $B_{m}^{i-1,1} \oplus \cdots \oplus B_{m}^{i-(m-1), m-1}$ inside $\mathcal{T}^{i}$ have a trivial intersection.

Proof. Elements of $\mathcal{T}^{i}$ are equivalence classes of $m$-tuples $\left(z_{0}, \ldots\right.$, $\left.z_{m-1}\right)$ in $A_{0}^{i 0} \oplus \cdots \oplus A_{0}^{i-(m-1), m-1}$, where $z_{0} \mapsto 0 \in A_{0}^{i+1,0}$ and $\operatorname{im}\left(z_{k}\right)$ $=\operatorname{im}\left(z_{k+1}\right) \in A_{0}^{i-k, k+1}$ for all $0 \leq k \leq m-2$.

The module $A_{m}^{i 0}$ comes from our filtration by rows, so its elements can be thought of as equivalence classes of elements $z \in A_{0}^{i 0}$ for which $z \mapsto 0 \in A_{0}^{i 1}$ and $z \mapsto 0 \in A_{0}^{i+1,0}$. Therefore, elements of $A_{m}^{i 0}$ map to $\mathcal{T}^{i}$ by $[z] \mapsto[(z, 0, \ldots, 0)]$.

Turning our attention to $B_{m}^{\bullet \bullet}$, the piece $B_{m}^{i-k, k}$ for each $1 \leq k \leq$ $m-2$, comes from the filtration by columns. This means it consists of equivalence classes of elements $w_{k} \in B_{0}^{i-k, k}$, where

$$
w_{k} \longmapsto 0 \in B_{0}^{i-k+1, k}
$$

and

$$
w_{k} \longmapsto \operatorname{im}\left(B_{0}^{i-k-1, k+1}\right) \subseteq B_{0}^{i-k, k+1},
$$

so we have an element $w_{k+1} \in B_{0}^{i-k-1, k+1}$ with $\operatorname{im}\left(w_{k}\right)=\operatorname{im}\left(w_{k+1}\right) \in$ $B_{0}^{i-k, k+1}$. Thus, the map $B_{0}^{i-k, k} \rightarrow \mathcal{T}^{i}$ is just 


$$
\left[w_{k}\right] \longmapsto\left[\left(0, \ldots, 0, w_{k}, w_{k+1}, 0, \ldots, 0\right)\right],
$$

where $w_{k}$ and $w_{k+1}$ appear in the $k$ th and $k+1$ th spots, respectively.

Our last module, $B_{m}^{i-(m-1), m-1}$ also comes from the filtration by columns, so its elements are equivalence classes of elements $w_{m-1} \in$ $B_{0}^{i-(m-1), m-1}$, where

$$
w_{m-1} \longmapsto 0 \in B_{0}^{i-(m-1)+1, m-1} .
$$

This means that our map $B_{m}^{i-(m-1), m-1} \rightarrow \mathcal{T}^{i}$ sends

$$
\left[w_{m-1}\right] \longmapsto\left[\left(0, \ldots, 0, w_{m-1}\right)\right] .
$$

Since $\operatorname{im}\left(A_{m}^{i 0}\right)$ has entries only in the 0th component, while the images of the $B_{m}^{i-k, k}$ s have entries only in the first through $(m-1)$ st components, it is clear that the images of $A_{m}^{i 0}$ and $B_{m}^{i-1,1} \oplus \cdots \oplus$ $B_{m}^{i-(m-1), m-1}$ have a trivial intersection.

Lemma 3.2 tells us that $\operatorname{im}\left(A_{m}^{i 0}\right) \subseteq \mathcal{T}^{i}$ is a submodule of $\operatorname{im}\left(\mathcal{T}^{i}\right) \subseteq$ $B_{m}^{i 0}$. Since, by Lemma 2.3, we know $\operatorname{Ass}_{R} B_{m-1}^{i 0}$ is finite, this means that $\operatorname{Ass}_{R} \operatorname{im}\left(A_{m}^{i 0}\right)$ is also finite. Therefore, $\operatorname{Ass}_{R} A_{m}^{i 0}$ is finite as well.

Now that we know $\operatorname{Ass}_{R} A_{m}^{i 0}$ is finite, we can repeatedly apply Lemma 2.2 to show that $\operatorname{Ass}_{R} A_{2}^{i 0}$ is finite. Since $A_{2}^{i 0} \cong H_{I}^{i}(R)$, this means $\operatorname{Ass}_{R} H_{I}^{i}(R)$ is finite for all $i$ as claimed.

The following corollary is a special case of the previous theorem whose assumptions are perhaps more familiar.

Corollary 3.3. Let $A$ be a finite-dimensional normal domain with an isolated singularity, where $A$ is finitely generated as an algebra over a field of characteristic 0 . If $R=A\left[t_{1}, \ldots, t_{\ell}\right]$ or $A\left[\left[t_{1}, \ldots, t_{\ell}\right]\right]$, then $\operatorname{Ass}_{R} H_{I}^{i}(R)$ is finite for any $i$ and any ideal $I$ of $R$.

Proof. Let $\mathfrak{m} \subset A$ be the maximal ideal which defines the nonsingular locus of $A$. Since $\operatorname{dim}(A)=m$ is finite, we know $\mathfrak{m}$ is generated, up to radical, by at most $m$ elements. Let $Y_{0}$ be the blow-up of $A$ along $\mathfrak{m}$. It is clear that $Y_{0}$ is covered by at most $m$ affine patches corresponding to the generators of $\mathfrak{m}$, and also that $\operatorname{depth}_{\mathfrak{m}} R \geq 2$ 
since ht $(\mathfrak{m}) \geq 2$ and $R$ is normal. Finally, we know that the higher cohomology of the structure sheaf of $A$ 's desingularization will consist of finitely generated $A$-modules supported only on the singular locus of $A$. Since Sing $(A)=\{\mathfrak{m}\}$, this means all higher cohomology of $Y_{0}$ is killed by some power of $\mathfrak{m}$, and hence is of finite length over $A$. Theorem 3.1 now implies that $\operatorname{Ass}_{R} H_{I}^{i}(R)$ is finite.

The only part of the proof of Theorem 3.1 which uses the fact that we are in characteristic 0 is the $D$-module theory needed for Lemma 2.2. (We also use $D$-module theory in the proof of Lemma 2.1, but this lemma is used only in the proof of Lemma 2.2, not in the proof of Theorem 3.1.) In equal characteristic $p>0$, Lyubeznik and others have successfully used the theory of $F$-modules to control the local cohomology of regular local rings in a way analogous to the use of $D$ modules in characteristic 0 , see [5]. Since many results about local cohomology which are proved in characteristic 0 using $D$-modules can be proved in equal characteristic $p>0$ using $F$-modules, it would be interesting to see if Lemma 2.2 can be proven using $F$-module theory. If it were possible, this would extend Theorem 3.1 to the equal characteristic $p$ case.

\section{REFERENCES}

1. M.P. Brodmann and R.Y. Sharp, Local cohomology: An algebraic introduction with geometric applications, Cambridge University Press, Cambridge, 1998.

2. W. Bruns and J. Herzog, Cohen-macaulay rings, Cambridge University Press, Cambridge, 1998.

3. C. Huneke and R. Sharp, Bass numbers of local cohomology modules, Trans. Amer. Math. Soc. 339 (1993), 765-779.

4. G. Lyubeznik, Finiteness properties of local cohomology modules (An application of d-modules to commutative algebra), Invent. Math. 113 (1993), 41-55.

5. F-modules: Applications to local cohomology and d-modules in characteristic $p>0$, J. reine angew. Math. 491 (1997), 65-130.

6. H. Matsumura, Commutative algebra, W.A. Benjamin, Inc., New York, 1970.

7. H. Robbins, Associated primes of local cohomology after adjoining indeterminates, J. Pure Appl. Alg. 218 (2014), 2072-2080.

Roanoke College, 221 College lane, Salem, VA 24153

Email address: robbinshr@gmail.com 